\title{
EXPLORATION OF POSITIVE MENTAL HEALTH LEVELS AMONG PROFESSIONALS IN PAKISTAN: AN INDIGENOUS PERSPECTIVE
}

\author{
Nadia Batool ${ }^{\mid 凶}$, Rubina Hanif
}

\begin{abstract}
OBJECTIVE: To investigate prevalence of mental health states (positive mental wellbeing levels) across professional groups working in diverse work settings in indigenous context.

METHODS: This cross-sectional study was conducted at public tertiary care hospitals (Jinnah Hospital Lahore, Combined Military Hospital), telecommunication companies (Warid, Telenor), Multinational firms, consultancy firms, Askari bank, Muslim Commercial Bank located at Islamabad and Lahore, Pakistan from February 2016 to December 2016. Data was collected from professionals $(\mathrm{N}=509)$ through purposive sampling technique. The present research employed Mental Health Continuum Short Form (MHCSF) and Brief Symptom Inventory (BSI) for measuring positive mental wellbeing levels and psychological distress (complete mental health).
\end{abstract}

RESULTS: Descriptive statistics revealed satisfactory psychometrics of MHCSF $(\alpha=0.86)$ and BSI $(\alpha=0.97)$ and its subscale results of ANOVA showed significant mean difference across professional groups in social wellbeing $F$ $(4,616=4.20 ; p<.001)$, somatization $F(4,566=4.58 p<0.01), \eta^{2}=0.03$, interpersonal sensitivity $F(4,599)=5.23 ; \mathrm{p}<0.001$ and paranoid ideation $F$ $(4,585=2.88 ; p<.02)$. Post-hoc analysis using Tukey followed by ANOVA further revealed mean differences between specific professional groups on social wellbeing dimension i.e., bankers and telecom officers $(-1.89 ; p<0.03)$, doctors and telecom officers $(-3.07 ; p<05)$.

CONCLUSION: Higher level of positive mental health buffers impact of negative stressors in personal and work domains. This also reflects a dire need to shift focus towards engaging mental health promotion strategies in order to develop flourishing, productive workforce, rather than solely focusing on assuaging mental illness and psychological distress.

KEY WORDS: Mental Health (MeSH); Positive mental health levels (Non$\mathrm{MeSH}$ ); Moderate mental health (Non-MeSH); Psychological distress (Non$\mathrm{MeSH}$ ); Flourishing (Non-MeSH); Languishing (Non-MeSH).

THIS ARTICLE MAY BE CITED AS: Batool N, Hanif R. An exploration of positive mental health levels among professionals in Pakistan: an indigenous perspective. Khyber Med Univ J 20I9;II(3):|45-5I. DOI: 10.35845/kmuj.2019.19409

\section{INTRODUCTION}

G lobally, exploration of positive dimensions of mental health has been invigorated. The current developments within the field of mental health lead to an all-inclusive definition of positive mental health encompassing wellbeing dimensions i.e. emotional, psychological and social well-being. Previously mental wellbeing term had been equated with absence of disease or infirmity. These current advances within conceptualization of positive wellbeing recognized it as measurable dimension distinct from psychological illness ${ }^{1,2}$ grounded in a social determinants health model supporting
I. National Institute of Psychology, Centre of excellence, Quaid-I-Azam University, Islamabad, Pakistan.

Email『:nadbatool@gmail.com

Contact \#: +92-323-528III15

$\begin{array}{ll}\text { Date Submitted: } & \text { June } 28,2019 \\ \text { Date Revised: } & \text { August I I, 2019 } \\ \text { Date Accepted: } & \text { August I3, 2019 }\end{array}$

populace health promotion. ${ }^{3,4}$ In recent times, World Health Organization (WHO) defined mental health as a "state of well-being in which an individual realizes his or her own abilities, can cope with the normal stresses of life, can work productively and is able to make a contribution to his or her community". 5 This led to an increased recognition of mental health as an integral part of the over health and wellbeing. The concept of well-being along with basic subcomponents of positive mental health has been illustrated comprehensively by hedonic and eudiamonic tradition. ${ }^{6}$ The hedonic tradition relates to emotional and cognitive appraisal of life satisfaction and positive affectivity. However, eudiamonic perspective captures individual's potential that fuels fulfillment and peak functioning. Positive mental health is theorized as encompassing wellbeing dimensions i.e. emotional, psychological and social well-being ${ }^{7,8}$, while integrating both approaches. The dual-continua model advocates presence of two related yet distinct continua representing mental health and mental dysfunction. ${ }^{9,10}$ Consequently effective interventions aimed at enhancing wellbeing can alter positive mental health levels, even though there were no indications of psychological illness.

Empirical literature has considered positive mental health as a protective factor" , as gains in positive indicators of wellbeing i.e. positive mental health anticipated deterioration in occurrence and prevalence of mental illness. Conversely deterioration in positive mental health levels envisages upsurge in occurrence and prevalence of mental illness. ${ }^{12}$ Some aspects of positive mental health are found to be linked with positive affectivity yielding higher 
TABLE I: POSITIVE MENTAL HEALTH THREE CATEGORIES DIAGNOSIS OF POSITIVE MENTAL HEALTH ON DESIGNATIONS $(n=509)$

\begin{tabular}{|l|c|c|c|c|}
\hline \multirow{2}{*}{$\begin{array}{l}\text { Positive Mental } \\
\text { Health Categories }\end{array}$} & \multicolumn{4}{|c|}{ Professional Categories } \\
\cline { 2 - 5 } & $\begin{array}{c}\text { Healthcare } \\
\text { Professionals } \\
(\mathbf{n}=95)\end{array}$ & $\begin{array}{c}\text { Bankers } \\
(\mathbf{n}=129)\end{array}$ & $\begin{array}{c}\text { Telecom } \\
\text { Officers } \\
(\mathbf{n}=\mathbf{2 9})\end{array}$ & $\begin{array}{c}\text { Consultants } \\
(\mathbf{n}=\mathbf{2 5 6})\end{array}$ \\
\hline Moderate mental health & $46(48.4 \%)$ & $62(48.1 \%)$ & $8(27.6 \%)$ & $49(19.1 \%)$ \\
\hline Flourishing & $36(37.9 \%)$ & $55(42.6 \%)$ & $15(51.7 \%)$ & $52(20.3 \%)$ \\
\hline Languishing mental health & $13(13.7 \%)$ & $12(9.3 \%)$ & $6(20.7 \%)$ & $155(60.6 \%)$ \\
\hline
\end{tabular}

level of life satisfaction and global functioning. ${ }^{13}$ In addition, lower levels of positive mental health increase propensity towards developing severe psychological dysfunction symptom manifestations e.g. depression, anxiety and stress across various cultures. ${ }^{14}$ Workplace environment has been recognized as key feature that determine employees mental health and overall wellbeing. Work environment has been increasingly recognized for buffering mental health and mental illness experienced by workforce through both promoting or hindering mental wellness and mental dysfunction. Recently, health care professional, bankers, financial consultants own mental health has been a focus of research worldwide. It has been recognized that high occupational stress, high work demands, low work control, high emotional demands, personal and organizational factors had a detrimental impact on mental health states of professionals working in health care and other fields. ${ }^{15}$

Across the globe, empirical studies reported higher prevalence of burnout among mental health professionals ${ }^{16}$, due to work environment while emphasizing role of sociodemographic variables such as age, gender, marital status and staff position along with job satisfaction to significantly impact mental health. Likewise, results of recent study ${ }^{17}$ showed high level of stress and burnout experienced by staff members to be linked with low level of job satisfaction, high turnover rate, and reduced work efficiency. ${ }^{18-21}$ Health care professionals, financial consultants, bankers due to highly demanding nature of their work, long working hours are more vulnerable towards experiencing mental strain causing anxiety and depression.

Within indigenous context, studies ${ }^{17}$ confirmed factors such as job insecurity, work schedules, pay package, job description to be significantly related to higher level of psychological distress, job dissatisfaction among doctors and health care providers. Another study ${ }^{22}$ conducted at tertiary care hospitals located at Lahore explored the existence of hidden stress among health care professionals, found significant impact of gender, education, service years, working hours and socioeconomic status. These factors were revealed to have an impact on psychological distress experienced by one-third of the doctors. Earlier studies conducted in Karachi reported that $25 \%$ to $30 \%$ of population was suffering from mental dysfunction of some sort such as anxiety and depression. ${ }^{23}$ While specifically higher vulnerability about two folds was reported among family practitioners and physicians for developing anxiety, depression even suicidal ideation. ${ }^{24}$ However, effective screening of doctors, other professionals is not possible with these scant evidences.

Given, mental health from positive perspective has emerged as an understudied area of investigation specifically with regard to diverse workforce. In this regard, exploration of positive mental health would yield insight in the light of new developments within mental health field i.e. dual continua model of positive mental health which considers mental health

TABLE II: COMPARISON OF POSITIVE MENTAL HEALTH LEVELS AND PSYCHOPATHOLOGY ACROSS PROFESSIONAL CATEGORIES $(n=509)$

\begin{tabular}{|c|c|c|c|c|c|c|c|c|c|c|c|c|}
\hline \multirow[t]{2}{*}{ Variables } & \multirow[b]{2}{*}{$a$} & \multicolumn{2}{|c|}{$\begin{array}{l}\text { Healthcare } \\
\text { professionals } \\
(\mathrm{n}=95)\end{array}$} & \multicolumn{2}{|c|}{$\begin{array}{l}\text { Bankers } \\
(\mathrm{n}=129)\end{array}$} & \multicolumn{2}{|c|}{$\begin{array}{l}\text { Telecom } \\
\text { officers } \\
(n=29)\end{array}$} & \multicolumn{2}{|c|}{$\begin{array}{l}\text { Consultants } \\
(n=256)\end{array}$} & \multirow[b]{2}{*}{$F$} & \multirow[b]{2}{*}{$p$} & \multirow[b]{2}{*}{$\eta^{2}$} \\
\hline & & Mean & SD & Mean & SD & Mean & SD & Mean & SD & & & \\
\hline Emotional wellbeing & 0.84 & 12.40 & 3.99 & 12.76 & 3.24 & 12.96 & 3.15 & 12.85 & 3.46 & 1.46 & 211 & - \\
\hline Psychological wellbeing & 0.82 & 25.56 & 5.76 & 26.34 & 5.79 & 25.89 & 6.77 & 26.89 & 5.09 & 1.33 & 0.257 & - \\
\hline Social wellbeing & 0.76 & 19.03 & 5.02 & 17.42 & 4.95 & 15.96 & 3.22 & 18.03 & 5.19 & 4.20 & 0.002 & 0.02 \\
\hline Somatization & 0.88 & 16.25 & 7.38 & 16.81 & 8.28 & 22.92 & 8.88 & 16.12 & 7.86 & 4.58 & 0.001 & 0.03 \\
\hline Obsessive compulsive & 0.82 & 15.86 & 5.96 & 15.65 & 6.64 & 16.66 & 3.30 & 15.47 & 6.05 & .496 & 0.739 & - \\
\hline Interpersonal sensitivity & 0.79 & 13.12 & 2.67 & 13.88 & 1.86 & 14.96 & 1.88 & $13.6 \mid$ & 2.09 & 5.23 & 0.000 & 0.01 \\
\hline Depression & 0.84 & 18.42 & 2.45 & 18.60 & 2.54 & 19.62 & 2.00 & 18.58 & 2.62 & 1.33 & 0.254 & - \\
\hline Anxiety & 0.84 & 18.54 & 2.77 & 18.76 & 2.52 & 18.11 & 2.18 & 18.40 & 2.61 & 1.60 & 0.173 & - \\
\hline Phobic anxiety & 0.95 & 12.08 & 5.78 & 11.21 & 5.51 & 12.58 & 4.89 & 11.34 & 5.96 & .60 & 0.658 & - \\
\hline Paranoid ideation & 0.77 & 16.15 & 2.40 & 16.14 & 2.14 & 17.35 & 1.90 & 16.64 & 1.88 & 2.88 & 0.022 & 0.01 \\
\hline Hostility & 0.89 & 16.00 & 2.63 & 16.00 & 2.21 & 16.62 & 2.61 & 16.40 & 2.40 & 0.968 & 0.424 & - \\
\hline Psychoticism & 0.77 & 14.94 & 2.30 & 15.07 & 2.26 & 15.78 & 2.11 & 14.94 & 2.30 & 0.964 & 0.427 & - \\
\hline
\end{tabular}


TABLE III: POST-HOC ANALYSIS OF PROFESSIONAL CATEGORIES ON POSITIVE MENTAL HEALTH AND PSYCHOPATHOLOGY $(n=509)$

\begin{tabular}{|c|c|c|c|c|c|c|}
\hline $\begin{array}{l}\text { Dependent } \\
\text { Variable }\end{array}$ & $\begin{array}{l}\text { (I) Professional } \\
\text { category }\end{array}$ & $\begin{array}{l}\text { (J) Professional } \\
\text { category }\end{array}$ & $\begin{array}{l}\text { Mean Difference } \\
(I-J)\end{array}$ & S. E & LL & UL \\
\hline \multirow{12}{*}{ Social well being } & \multirow{3}{*}{ Bankers } & Telecom & 1.474 & 1.04 & -1.38 & 4.33 \\
\hline & & Doctors & -1.603 & 0.699 & -3.51 & 0.310 \\
\hline & & Consultants & .651 & 0.555 & -2.17 & 0.868 \\
\hline & \multirow{3}{*}{ Telecom } & Bankers & -1.474 & 1.04 & -4.33 & 1.38 \\
\hline & & Doctors & $-3.078 *$ & 1.08 & -6.04 & -0.116 \\
\hline & & Consultants & -2.126 & 0.995 & -4.85 & 0.598 \\
\hline & \multirow{3}{*}{ Doctors } & Bankers & 1.60 & 0.699 & -0.310 & 3.51 \\
\hline & & Telecom & 3.078* & 1.08 & 0.116 & 6.04 \\
\hline & & Consultants & 0.952 & 0.621 & -0.747 & 2.65 \\
\hline & \multirow{3}{*}{ Consultants } & Bankers & 0.651 & 0.555 & -0.868 & 2.17 \\
\hline & & Telecom & 2.126 & 0.995 & -0.598 & 4.85 \\
\hline & & Doctors & -.952 & 0.621 & -2.65 & 0.747 \\
\hline \multirow{9}{*}{ Somatization } & \multirow{3}{*}{ Bankers } & Telecom & $-6.11 * *$ & 1.68 & -10.7 & $-|.5|$ \\
\hline & & Doctors & 0.558 & 1.10 & -2.477 & 3.59 \\
\hline & & Consultants & 0.684 & 0.881 & -1.72 & 3.09 \\
\hline & \multirow{3}{*}{ Telecom } & Bankers & $6.11 * *$ & 1.68 & 1.51 & 10.7 \\
\hline & & Doctors & $6.67 * *$ & 1.74 & 1.90 & 11.4 \\
\hline & & Consultants & $6.79 * *$ & 1.60 & 2.40 & 11.1 \\
\hline & \multirow{3}{*}{ Consultants } & Bankers & -0.558 & 1.10 & -3.59 & 2.47 \\
\hline & & Telecom & $-6.67 * *$ & 1.74 & -11.44 & -1.90 \\
\hline & & Consultants & .126 & 0.991 & -2.58 & 2.83 \\
\hline \multirow{12}{*}{ Interpersonal sensitivity } & \multirow{3}{*}{ Bankers } & Telecom & -1.07 & $0.44 I$ & -2.28 & 0.133 \\
\hline & & Doctors & 0.760 & 0.293 & -0.041 & 1.56 \\
\hline & & Consultants & 0.274 & 0.233 & -0.364 & 0.914 \\
\hline & \multirow{3}{*}{ Telecom } & Bankers & 1.07 & 0.44193 & -0.133 & 2.28 \\
\hline & & Doctors & $1.83 * *$ & 0.45670 & 0.586 & 3.08 \\
\hline & & Consultants & $1.35 *$ & 0.42106 & 0.198 & 2.50 \\
\hline & \multirow{3}{*}{ Doctors } & Managers & -0.760 & 0.29306 & -1.56 & 0.041 \\
\hline & & Telecom & $-1.83 * *$ & 0.45670 & -3.08 & -0.586 \\
\hline & & Consultants & -0.486 & 0.26053 & -1.1989 & 0.226 \\
\hline & \multirow{3}{*}{ Consultants } & Bankers & -0.274 & 0.23367 & $-0.9 \mid 40$ & 0.364 \\
\hline & & Telecom & $-1.35 * *$ & 0.42106 & -2.5025 & -0.198 \\
\hline & & Doctors & 0.486 & 0.26053 & -0.2268 & 1.19 \\
\hline \multirow{12}{*}{ Paranoid ideation } & \multirow{3}{*}{ Bankers } & Telecom & -10.21 & 0.44454 & -2.4284 & 0.004 \\
\hline & & Doctors & -0.007 & 0.29235 & -0.8070 & 0.792 \\
\hline & & Consultants & -0.50214 & 0.23480 & -1.1446 & 0.1404 \\
\hline & \multirow{3}{*}{ Telecom } & Bankers & 1.21198 & 0.44454 & -0.0044 & 2.4284 \\
\hline & & Doctors & I.20497 & 0.45856 & -0.0498 & 2.4597 \\
\hline & & Consultants & 0.70984 & 0.42420 & -0.4509 & 1.8706 \\
\hline & \multirow{3}{*}{ Doctors } & Mangers & 0.00701 & 0.29235 & -0.7929 & 0.8070 \\
\hline & & Telecom & -1.20497 & 0.45856 & -2.4597 & 0.0498 \\
\hline & & Consultants & -0.49513 & 0.26037 & -1.2076 & 0.2173 \\
\hline & \multirow{3}{*}{ Consultants } & Managers & 0.50214 & 0.23480 & -0.1404 & 1.1446 \\
\hline & & Telecom & -0.70984 & 0.42420 & -1.8706 & 0.4509 \\
\hline & & Doctors & 0.49513 & 0.26037 & -0.2173 & 1.2076 \\
\hline
\end{tabular}

${ }^{*} \mathrm{p}<0.05,{ }^{* *} \mathrm{p}<0.01$

and mental illness along two continua which are moderately negatively correlated with each other. This also allows for combined assessment of both positive and negative indicators of mental wellbeing (complete mental health) along with categorical diagnosis of mental health levels (flourishing, moderate mental health \& languishing mental health). Since mental health levels directly relate to optimal or less than optimal functioning states, scant 
empirical evidence stresses the need to bridge existing gap in indigenous literature by exploring positive mental health dimensions i.e. emotional, psychological and social well-being comprehensively in general public as well as across professional groups. This study was conducted to investigate prevalence of mental health states (positive mental wellbeing levels) across professional groups working in diverse work settings in indigenous context.

\section{METHODS}

This cross sectional study was conducted at public tertiary care hospitals (Jinnah Hospital Lahore, Combined Military Hospital), telecommunication companies (Warid telecom, Telenor), Multinational firms, consultancy firms, Askari, bank, Muslim Commercial Bank located at Islamabad and Lahore, Pakistan from February 2016 to December 2016. Participants were included by utilizing purposive convenient sampling technique. A sample comprised of $(\mathrm{N}=$ $509)$ employed men $(n=30 \mathrm{I}) 59.14 \%$, women $(n=208) 40.86 \%$ with age range 24-64. Their work experience ranged from I to 40 years. Their average age was $(M=30.72$ years, $S D=7.03)$. The inclusion criteria entailed minimum six months of experience in respective organization. The current study seeked ethical approval from the advanced board of research studies of Quaid-I-Azam University. Informed consent was taken from study participants.

Metal health continuum short form (MHC-SF) comprised of 14 theoretically derived items, representing three dimensions of well-being i.e. emotional (hedonic), psychological and social wellbeing. Emotional wellbeing dimension was measured by items reflecting individual's level of positive affectivity and cognitive evaluations of life satisfaction. While psychological wellbeing dimensions was measured by six items based on Ryff (1989) theorization revealing individual's peak functioning levels ensuring selfactualization. Furthermore, social wellbeing was measured by five items encompassing individual's social functioning domains. MHC-SF (six-point) Likert scale had response options ranged from ('never', 'once or twice', 'about once a week', '2 or 3 times a week', 'almost every day', or 'every day'). ${ }^{13}$

Besides measuring global mental wellbeing, MHC-SF also allows for categorical diagnosis of mental health levels i.e., flourishing, moderate and languishing mental health. Individuals who endorsed "everyday" and "almost every day" on three of the emotional wellbeing items and minimum six of the eleven items of positive functioning were characterized as having flourishing mental health. On the other hand, individuals who endorsed (never or once or twice) at least one of emotional wellbeing item along with low scoring on six dimensions of positive functioning were characterized to have languishing mental health. Those individuals who did not meet criteria for flourishing and moderate mental health category were designated to have moderate mental health level.

Brief symptom inventory (BSI) had been used for screening psychopathology. ${ }^{25}$ It consisted of 53 items, having nine subscales (depression, anxiety, phobic anxiety, interpersonal sensitivity, obsessive-compulsive disorder, hostility, paranoid ideation, psychoticism, \& somatization). All nine subscales measured different dimensions of mental illness. Each item of individual psychological distress was responded on 6 point response options ranging from not at all $=1$, a little bit $=2$, moderately $=3$, quite a bit $=4$, extremely $=5$, Refuse to answer $=6$. The study participants were approached in their prospective work organizations. The purpose of study was explained and informed consent was taken from the participants. After seeking informed consent, questionnaire booklet were handed over to them. Both validated instruments i.e., MHC-SF and BSI were used for measuring study variables. Permission was sought for use in our culture from the original authors of these measures. These instruments MHC-SF and BSI had been administered primarily for tryout on professionals for checking their suitability and item relevance with regard to indigenous organizational context. The results of tryout assured that these measures were suitable for use in our culture.

The data was entered on SPSS version 20 and cross validated by random checking. Frequency and percentages of mental health levels across professionals groups were computed. A p-value of $<0.05$ was considered significant for interpreting mean differences on positive mental health and psychological distress across professional categories.

\section{RESULTS}

Out of 550 distributed questionnaire booklets, 509 professionals responded. Amongst these 509 respondents, there were $(n=95)$ health care professionals, $(n=129)$ bankers, $(n=29)$ telecom officers and $(n=256)$ consultants. The study participants were working in their specialized filed in numerous organizations i.e., Jinnah Hospital Lahore, Combined Military hospital, Telenor, Mobilink, Askari Bank, Muslim Commercial Bank (Lahore), located at Islamabad and Lahore, Pakistan. The descriptive of the study variables revealed satisfactory psychometrics for MHC-SF $(\alpha=0.86)$ total and its subscales i.e., emotional wellbeing $(\alpha=0.84)$, psychological wellbeing $(\alpha=0.82)$ and social wellbeing $(\alpha=0.76)$ and brief symptom inventory total $(\alpha=0.97)$ and subscales. Both measures MHC-SF and BSI had shown good reliability and validity for assessment of positive mental wellbeing and psychological dysfunction. The present data met the assumptions of normality, values of skewness and kurtosis were found within acceptable range.

Frequencies and percentages of mental health level were computed for exploring prevalence of flourishing, moderate and languishing mental health levels across professional groups. The findings of present study indicated bankers to have highest proportion of flourishing mental health level followed by consultants, doctors, telecom officers (Table I). Moreover, Analysis of Variance (ANOVA) was carried out for analyzing mean differences in positive mental health (MHC-SF, measure of positive mental health) and psychopathology (BSI, measure of psychopathology) across professional categories. Results of ANOVA (Table II) showed significant mean difference across professional groups in social wellbeing $F(4,616=4.20$; $\mathrm{p}<.00 \mathrm{I})$, somatization $\mathrm{F}(4,566=4.58$; $\mathrm{p}<0.0 \mathrm{I}), \eta^{2}=0.03$, interpersonal sensitivity $F(4,599=5.23 ; p<0.00 I)$ and paranoid ideation $\mathrm{F}(4,585=2.88 ; \mathrm{p}<.02)$ 
(Table II). Post-hoc analysis using Tukey (Table III) followed by ANOVA further revealed mean differences between specific professional groups on social wellbeing dimension i.e., bankers and telecom officers (-I.89; $\mathrm{p}<0.03)$, doctors and telecom officers $(-3.07$; $\mathrm{p}<05)$. Similarly, significant mean difference were also found between Bankers and telecom officers (6.1I; $p<0.05)$, doctors and telecom officers (6.67; $\mathrm{p}<0.00 \mathrm{I})$, telecom officers and consultants $(6.79 ; \mathrm{p}<0.00 \mathrm{I})$ on somatization subscale of BSI. For interpersonal sensitivity, mean differences were found significant between telecom officers and doctors $(1.83 ; \mathrm{p}<0.0 \mathrm{I})$, telecom officers and consultants $(\mathrm{I} .35 ; \mathrm{p}<0.0 \mathrm{I})$. However, mean difference were found significant between mangers and telecom officers on paranoid ideation $(1.21 ; \mathrm{p}<0.05)$.

\section{DISCUSSION}

In recent decades, the term mental health is conceptualized as presence of certain positive indicators that ascertain high level of social, emotional and psychological wellbeing (optimal functioning of the individual). These developments within the conceptualization of mental health allows for comprehensive evaluation of individual functioning levels in personal and social realms. Keyes' polythetic approach for classifying flourishing mental health entails presence of high levels of emotional, psychological and social wellbeing that leads towards high level of efficiency and productivity. Mental health states diagnosis was modeled after the DSM-III-R approach to detecting MDE. ${ }^{13}$ According to Keyes, positive mental health signifies presence of clusters of positive indicators related to both affectivity and functioning (Syndrome of positive affectivity) in similar way, DSM-R (cluster of symptoms) proposed presence of specific set of symptoms for diagnosing Major Depression. Cross culturally Keyes model of mental health has generated support across varied nationalities e.g. US MIDUS data ${ }^{2}$, U.S. adolescent, Dutch adults $^{12}$ and Setswana-speaking SouthAfrican adults. ${ }^{26}$

Globally, industrialized countries have experienced substantial shifts with regard to competitiveness, mergers, downsizing, and turbulence of organizational demands along with fast paced technological evolution that all has led to increased vulnerability towards psychological strain affecting the mental health of diverse workforce. Evidence highlight progressive deterioration of working conditions and subjective wellbeing among European countries. ${ }^{27}$ Over the last decade, the steady rise in mental ill health issues among health care personnel and professionals working in diverse organizations seems challenging despite presence of a strong social security system in developed countries. Moreover, shift towards excessive work load, imbalance between demand and resources, service jobs, lack of personal autonomy in making decisions, and increasing computerized tasks have significantly augmented susceptibility towards sharp rise in psychological and mental health problems. ${ }^{28,29}$ Empirical literature in developed countries (e.g. United States, United Kingdom, Canada, New Zealand \& Switzerland) has also shown high incidence of depression (74\% South African workforce) bipolar disorder, burn out, anxiety disorders, high suicide rates three times more prevalent in general practitioners and surgeons as compared to general population. ${ }^{30}$ At workplace mental health problems like depression, anxiety, high stress levels leads to serious consequences for both individual's wellbeing and organizational outcomes.

In accord with these findings, numerous studies that explored variables of psychological distress, anxiety depression among organizational employees, doctors reported similar trends within indigenous context. ${ }^{17,20-22}$ The lack of the sound infrastructure of hospitals, excessive work load, long hours of duty, exposure to physical attacks and poor work conditions make it difficult for health care professionals to pursue their responsibilities in a fully efficient way as a flourishing workforce. The present study found that $36 \%$ of the health care professionals were having flourishing mental health level, while $46 \%$ came within moderate mental health category. However, Pakistan has dominant collectivistic culture where people enjoy high level of social connectivity and networking, keeping joint ventures superior to their individualistic goals." This might reflect highest frequency of flourishing mental health levels among mangers followed by consultants in present study though they were also exposed to stressors like long working hours, job insecurity, competitiveness, weak infrastructure causing instability and distress among employees. High level of emotional and psychological wellbeing while pronounced low level of social wellbeing among western nationalities has been found experience due to prevalent individualistic norms." On subscales of Brief Symptom Inventory somatization, interpersonal sensitivity and paranoid ideation telecom employees reported higher means reflecting high level of psychological distress experienced by employees working in telecom sector as compared to other professional categories.

Findings of the current study revealed lower percentage of health care professionals falling in flourishing mental health category in contrast to other professional groups. The mental wellbeing and optimal functioning of health care professionals gets affected due to the multiple occupational roles and sensitive nature of their work. Professional pursuits take a substantial toll on the mental capacities of health care professionals making them more vulnerable towards psychological distress instilling anxiety and depression compared to organizational workforce. Few studies conducted in indigenous context supports improved work environment lead to higher level of psychological wellbeing and motivation experienced by older medical professionals as compared to younger professionals.

Cross cultural studies using MHC-SF have reported wide variation in prevalence rates of flourishing from $8 \%$ among South Korean adults ${ }^{31}$ to $49 \%$ among US college students, ${ }^{28} 20 \%$ flourishing among adult South Africans, ${ }^{30}$ and $44 \%$ flourishing among Chinese adults. Moderate mental health entails functioning at level less than optimal leading to reduce an individual capacity to fully utilize their capability. Moreover, a considerable body of evidence exists to support the reliability of MHC-SF for predicting future risk of mental illness among adults ${ }^{5}$ and work-related productivity and health care use. ${ }^{29}$ Within indigenous context no published study 
has yet been affirmed to measure mental health levels across professional categories.

The limitation of the present study includes small sample size across professional groups. Some specific public and private organizations were included in the study which limits its scope for generalizability. The present study utilized original English version of the instruments, since Urdu translated versions were not used, this might have impacted findings, although sample comprised of literate professional populace. Future study should incorporate a more representative sample to build a strong nomothetic base for exploring mental health construct indigenously for gaining a more comprehensive and richer understanding.

In nutshell, the emergent epidemic of mental health issues has plagued the workforce leading to an upsurge in debilitating economic burden of disease and disabilities worldwide. ${ }^{32}$ There is strong need to improve the organizational structure and psychological environment for promoting optimal mental health to reduce the high prevalence of anxiety, depression, suicidal ideation among healthcare professionals and physicians. However, high level of psychological distress was also reported by telecom employees indicating a dire need for focusing on mental wellness of their employees. Given, there is strong need for adopting positive mental health promotion and protection approach rather than previous focus towards minimizing mental dysfunction and distress .Ample empirical evidence calls for utilizing ethno-specific approaches to mental health by mental health practionniers and institutions as these approaches allow for and adapt to dissimilarities across cultures. ${ }^{33}$ The current advancement in positive organizational behavior domain addresses mental health issues of employees by building strong psychological resources e.g. psychological capital to improve employee psychological health that reduces low productivity and high turnover problems faced by organizations. Thus, building mentally healthy work groups facilitate personnel wellbeing along with accomplishment of positive organizational outcomes.

\section{CONCLUSION}

Higher level of positive mental health buffers impact of negative stressors in personal and work domains. Prevention within the mental health field needs to shift focus towards enhancing positive qualities of daily functioning. Mental health promotion strategies need to be employed for rising flourishing, productive workforce. As prior focus solely on eliminating diseases and disability has not brought any positive change in elevating mental resilience and also fostering positive organizational outcomes.

\section{REFERENCES}

I. Magyary D. Positive mental health: A turn of the century perspective. Int J Ment Health Nurs 2002 Jan I; 23(4):33I-49. DOI: I0.1080/ $016 \mid 2840290052550$.

2. Keyes CL. Mental illness and/or mental health? Investigating axioms of the complete state model of health. J Consult Clin Psychol 2005 Jun; 73(3):539-48. DOI: 10.1037/ 0022006X.73.3.539.

3. Huppert FA, Whittington JE. Evidence for the independence of positive and negative wellbeing: Implications for quality of life assessment. Br J Health Psychol 2003 Feb; 8(I): 107-22.

4. Keyes CL, Dhingra SS, Simoes EJ. Change in level of positive mental health as a predictor of future risk of mental illness. Am J Public Health 2010 Dec; I00(I 2):2366-7I.DOI: 10.2I05/AJPH.2010.192245.

5. World Health Organization. Mental Health Action Plan for Europe: Facing the Challenges, Building Solutions. Geneva, Switzerland: World Health Organization. 2005.

6. Keyes CL, Shmotkin D, Ryff CD. Optimizing well-being: The empirical encounter of two traditions. J Pers Soc Psychol 2002 Jun;82(6): 1 007-22.

7. Deci EL, Ryan RM. Facilitating optimal motivation and psychological well-being across life's domains. Can Psychol 2008 Feb;49(I): 14-23. DOI:10.1037/0708-559I.49.1.I4.

8. Keyes CL. The mental health continuum: From languishing to flourishing in life. J Health Soc Behav 2002;43(2);207-22.

9. Wang $X$, Zhang $D$, Wang J. Dualfactor model of mental health: Surpass the traditional mental health model. Psychology $201 \mathrm{I}$ Nov 4;2(08):767-72. DOI:10.4236/ psych.2011.28I17.

10. Keyes CL. Promoting and protecting mental health as flourishing: $A$ complementary strategy for improving national mental health. Am Psychol 2007 Feb;62(2):95-108. DOI: 10.1037/0003-066X.62.2.95.

II. Westerhof G], Keyes CL. Mental illness and mental health: The two continua model across the lifespan. J Adult Dev 2010 Jun I; I7(2): I 10-19. DOI: 10.1007/s 10804-009-9082-y.

12. Seow LSE, Vaingankar JA, Abdin E, Sambasivam $R$, Jeyagurunathan $A$, Pang $S$, et al. Positive mental health in outpatients with affective disorders: Associations with life satisfaction and general functioning. J Affect Disord 2016 Jan 15;190:499-507. DOI: 10.1016/j.jad.2015.10.021.

13. Schönfeld P, Brailovskaia J, Bieda A, Zhang XC, Margraf J. The effects of daily stress on positive and negative mental health: Mediation through self-efficacy. Int J Clin Health Psychol 2016 Jan;16(I):I-I0. DOI: 10.1016/j.jijchp.2015.08.005.

14. Thompson WT, Cupples ME, Sibbett $\mathrm{CH}$, Skan DI, Bradley T. Challenge of culture, conscience, and contract to general practitioners' care of their own health: qualitative study. $\mathrm{Br}$ Med J 200I Sep 29;323(73I5):728-3I. DOI: I0.1 |36/bmj.323.73।5.728.

15. Dowell AC, Hamilton S, McLeod DK. Job satisfaction, psychological morbidity and job stress among New Zealand general practitioners. N Z Med J 2000 Jul I4; I I 3( I I | 3): 269-72.

16. Ali BS, Rahbar MH, Naeem S, Tareen AL, Gul A, Samad L. Prevalence of and factors associated with anxiety and depression among women in a lower middle class semi-urban community of Karachi, Pakistan. J Pak Med Assoc 2002 Nov; 52(I I):5 I 3-7.

17. Khuwaja AK, Qureshi R, Andrades M, Fatmi Z, Khuwaja NK. Comparison of job satisfaction and stress among 
male and female doctors in teaching hospitals of Karachi. J Ayub Med Coll Abbottabad 2004; 16(I):23-7.

18. Atif K, Khan HU, Malik AF. Do doctors have hidden distress; a study conducted at tertiary care hospital at Lahore. J Pak Med Assoc 2016 Jan;66(I):63-7.

19. Atif K, Boghsani GT, Javed A, Javed S. Does lack of job satisfaction intensify psychological distress among clinicians, and vice versa? Electron J Gen Med 2018; I5(5):em82.

20. Khuwaja AK, Qureshi R, Azam SI. Prevalence and factors associated with anxiety and depression among family practitioners in Karachi, Pakistan. J Pak Med Assoc 2004 Feb;54(2):45-9.

21. Giorgi G, Arcangeli G, Perminiene M, Lorini C, Ariza-Montes A, Fiz-Perez], et al. Work-Related Stress in the Banking Sector: A Review of Incidence, Correlated Factors, and Major Consequences. Front Psychol 2017 Dec;8:2 I66. DOI: 10.3389/fpsyg.2017.02166.

22. Chatta HA, Zafar A, Amin F. Measurement of Stress among Doctors in Surgical Intensive Care Unit (ICU) of Lahore General Hospital, Lahore. Pak J Med Health Sci 20 I 7; I I (3):874-7.
23. Picco L, Yuan Q, Vaingankar JA, Chang S, Abdin E, Chua HC, et al. (2017) Positive mental health among health professionals working at a psychiatric hospital. PLoS One 2017 Jun $7 ; \mid$ 2(6):e0I78359. DOI: I0.1371/journal.pone.0178359.

24. Derogatis LR, Melisaratos N. The brief symptom inventory: an introductory report. Psychol Med 1983 Aug; | 3(3):595-605.

25. Keyes CL, Wissing M, Potgieter JP, Temane M, Kruger A, Van Rooy S. Evaluation of the mental health continuum-short form (MHC-SF) in Setswana speaking South Africans. Clin Psychol Psychother 2008 May; I5(3): I8I-92. DOI: I0. I002/cpp.572

26. Keyes CL, Grzywacz JG. Health as a complete state: The added value in work performance and healthcare costs. J Occup Environ Med 2005 May I;47(5):523-32. DOI: 10.1097/ $01 . j o m .0000161737 .21198 .3 \mathrm{a}$.

27. Keyes CLM. The nature and importance of positive mental health in America's adolescents. In: Gilman R, Huebner ES, Furlong KJ (Eds). Handbook of positive psychology in schools. 2009; pp: 9-23. Routledge / Taylor \& Francis Group, New York, USA.

28. Lim YJ, Ko YG, Shin HC, Cho Y.
Prevalence and correlates of complete mental health in the South Korean adult population. In Mental Well-Being 2013 (pp. 9l-109). Springer, Dordrecht.

29. Ox J. Understanding doctors' performance. Radcliffe Publishing; 2006.

30. Cappelli P, Bassi L, Katz H, Knoke D, Osterman P, Useem M. Change at work. New York: Oxford University Press; 1997.

31. Keyes CL, Eisenberg D, Perry GS, Dube SR, Kroenke K, Dhingra SS. The relationship of level of positive mental health with current mental disorders in predicting suicidal behavior and academic impairment in college students. J Am Coll Health 2012 Feb I;60(2):I26-33. DOI: I0.1080/0744848I.20II.608393

32. Robone $\mathrm{S}$, Jones $\mathrm{AM}, \mathrm{Ri}+$ ce $\mathrm{N}$. Contractual conditions, working conditions, health and well-being in the British household panel survey. Eur J Health Econ 201 I Oct; 12(5): 429-44. DOI: 10.1007/s10198-0I00256-0.

33. Llena-Nozal A. The impact of work status and working conditions on mental health in four OECD countries. Natl Inst Econ Rev 2009;209(I):72-87. DOI: 10.1177/0027950109345234

\section{AUTHORS' CONTRIBUTIONS}

Following authors have made substantial contributions to the manuscript as under:

NB: Acquisition, analysis \& interpretation of data, drafting the manuscript, final approval of the version to be published

RH: Concept and study design, critical review, final approval of the version to be published

Authors agree to be accountable for all aspects of the work in ensuring that questions related to the accuracy or integrity of any part of the work are appropriately investigated and resolved.

\begin{tabular}{|c|}
\hline CONFLICT OF INTEREST \\
Authors declared no conflict of interest \\
GRANT SUPPORT AND FINANCIAL DISCLOSURE \\
NIL \\
\hline
\end{tabular}

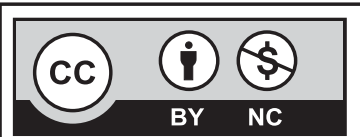

This is an Open Access article distributed under the terms of the Creative Commons Attribution-Non Commercial 2.0 Generic License. 